\title{
Performances of the Rapid Polymyxin Acinetobacter and Pseudomonas Tests for Colistin Susceptibility Testing
}

\author{
Mathilde Lescat, ${ }^{1-4}$ Laurent Poirel, $^{1,2,5}$ Aurélie Jayol,, ${ }^{1,2,5}$ and Patrice Nordmann ${ }^{1,2,5,6}$
}

\begin{abstract}
Objectives: Owing to the emergence of colistin resistance in nonfermenting Gram negative bacteria, reliable and rapid techniques for testing colistin susceptibility are needed. We evaluated the performances of the Rapid Polymyxin Acinetobacter and Pseudomonas tests using a collection of Acinetobacter baumannii and Pseudomonas aeruginosa clinical isolates.

Methods: Colistin susceptibility of A. baumannii and P. aeruginosa isolates (colistin susceptible and colistin resistant) was tested with the Rapid Polymyxin Acinetobacter and Pseudomonas tests and compared with the broth microdilution method.

Results: The Rapid Polymyxin Acinetobacter and Pseudomonas tests were able to detect all colistin-resistant and all colistin-susceptible $A$. baumannii and $P$. aeruginosa isolates within 4 hours.

Conclusion: The Rapid Polymyxin Acinetobacter and Pseudomonas tests are reliable techniques for detecting colistin resistance. Overall, both techniques allow an accurate and a rapid screening $(<4$ hours) of colistin resistance in A. baumannii and $P$. aeruginosa.
\end{abstract}

Keywords: susceptibility testing, rapid diagnostic test, polymyxin, Acinetobacter, Pseudomonas

\section{Introduction}

$A$ CINETOBACTER BAUMANNII AND Pseudomonas aeruginosa belong to the ESKAPE group of pathogens (Enterococcus faecium, Staphylococcus aureus, Klebsiella pneumoniae, Acinetobacter baumannii, Pseudomonas aeruginosa, and Enterobacter species) identified as the most important bacterial species in clinical settings as a source of multidrug resistance. ${ }^{1}$ Infections due to multidrug-resistant (MDR) A. baumannii and $P$. aeruginosa species, especially carbapenem-resistant isolates, are increasingly reported in health care facilities, being responsible for nosocomial infections that may lead to fatal outcomes due to limited therapeutic options. ${ }^{2-4}$ The Center for Diseases Control of Atlanta in the United States and then the World Health Organization classified the carbapenem-resistant $A$. baumannii and $P$. aeruginosa among the most serious pathogens exhibiting multidrug resistance. ${ }^{5,6}$ Consequently, old antibiotics such as polymyxins (colistin) are increasingly used as last resort treatment for treating MDR A. baumannii and $P$. aeruginosa. ${ }^{4,7}$ This highlights the importance of giving rapid results of polymyxin susceptibility to optimize the antibiotic stewardship.
The current standard method of detection for colistin susceptibility in Gram negatives is the manual determination of minimum inhibitory concentration (MIC) by the broth microdilution (BMD) method. ${ }^{8}$ However, this procedure is technician dependent (partly due to the fact that colistin must be weighted for each experiment), is time consuming, and results are obtained in 24 hours.

Recently, Nordmann et al. developed the Rapid Polymyxin Nordmann-Poirel (NP) test that categorizes colistinsusceptible from colistin-resistant enterobacterial isolates in $<2$ hours. ${ }^{9}$ However, this test based on visualization of glucose metabolization cannot be applied to nonfermenting Gram negative bacteria, such as $A$. baumannii and $P$. aeruginosa.

Using the same principle as the Rapid Polymyxin NP test, the Rapid Polymyxin Acinetobacter and Pseudomonas tests have recently been developed by Elitech Microbiology (www.elitechgroup.com/france) and are liquid-based techniques that rely on the colorimetric detection of a rapid metabolism related to bacterial growth, in the presence of a defined concentration of colistin. The acidification of the medium related to bacterial growth is visualized by the color shift of the $\mathrm{pH}$ indicator (red to yellow or orange with the red phenol in the Rapid Polymyxin Acinetobacter test and

\footnotetext{
${ }^{1}$ Emerging Antibiotic Resistance Unit, Medical and Molecular Microbiology, Department of Medicine, Faculty of Science and Medicine, University of Fribourg, Fribourg, Switzerland.

${ }^{2}$ INSERM European Unit (LEA, IAME), Paris France.

${ }^{3}$ Université Paris Nord, Sorbonne Paris Cité, Paris, France.

${ }^{4}$ AP-HP, Hôpitaux Universitaires Paris Seine Saint-Denis, Paris, France.

${ }^{5}$ Swiss National Reference Center for Emerging Antibiotic Resistance (NARA), University of Fribourg, Fribourg, Switzerland.

${ }^{6}$ Institute of Microbiology, University of Lausanne and University Hospital Center, Lausanne, Switzerland.
} 
green to violet with bromocresol purple in the Rapid Polymyxin Pseudomonas test).

The objective of this study was to evaluate the performance of those novel tests by comparison with the BMD method using a collection of colistin-susceptible and colistinresistant $A$. baumannii and $P$. aeruginosa clinical isolates.

\section{Materials and Methods}

\section{Bacterial strains}

This study was carried out using 38 clinical isolates of A. baumannii $(n=21)$ and $P$. aeruginosa $(n=17)$ identified the species level using the Microflex benchtop matrix assisted laser desorption ionization-time of flight (MALDI-TOF) mass spectrometer (Brücker, Champs-sur-Marne, France). Nine out of the 21 A. baumannii isolates and 10 out of the 17 P. aeruginosa isolates were susceptible to colistin. Twelve out of the $21 \mathrm{~A}$. baumannii and 7 out of the 17 P. aeruginosa isolates were colistin resistant according to BMD testing. Isolates were grown on Luria Bertani (LB; Sigma, Saint Louis, MO) agar plates at $35^{\circ} \mathrm{C} \pm 2^{\circ} \mathrm{C}$ for 18 hours. The colistin-susceptible strain P. aeruginosa ATCC 27853 and the colistin-resistant Escherichia coli R2739 were used as negative and positive controls, respectively, for the determination of MIC of colistin by the BMD method. None of the colistin-resistant isolates carried a plasmid-encoded MCR-like (MCR-1 to -4) colistin resistance determinant, as assessed by the negative polymerase chain reaction results (data not shown).

\section{Susceptibility testing}

The BMD method was performed in triplicate and interpreted according to the EUCAST/CLSI joined guidelines ${ }^{8}$ as described. ${ }^{10}$

Table 1. Minimum Inhibitory Concentrations of Colistin (mg/L) Using the Broth Microdilution Method and Results of the Rapid Polymyxin Acinetobacter and Pseudomonas Tests

\begin{tabular}{|c|c|c|c|c|c|c|}
\hline \multirow[b]{2}{*}{ Isolate } & \multirow[b]{2}{*}{ Species } & \multirow[b]{2}{*}{ Origin } & \multirow[b]{2}{*}{ Phenotype } & \multirow{2}{*}{$\begin{array}{c}B M D \\
M I C \text { colistin } \\
(m g / L)\end{array}$} & \multicolumn{2}{|c|}{$\begin{array}{c}\text { Rapid Polymyxin Acinetobacter/ } \\
\text { Pseudomonas test }\end{array}$} \\
\hline & & & & & $\begin{array}{l}\text { MIC colistin } \\
(m g / L)\end{array}$ & Discrepancies $^{\mathrm{a}}$ \\
\hline FR-242 & Acinetobacter baumannii & Switzerland & $\mathrm{S}$ & $<0.12$ & $\leq 2$ & No \\
\hline FR-243 & A. baumannii & Turkey & $\mathrm{S}$ & $<0.12$ & $\leq 2$ & No \\
\hline FR-244 & A. baumannii & Turkey & $\mathrm{S}$ & $<0.12$ & $\leq 2$ & No \\
\hline FR-245 & A. baumannii & Turkey & $\mathrm{S}$ & $<0.12$ & $\leq 2$ & No \\
\hline FR-246 & A. baumannii & Turkey & $\mathrm{S}$ & $<0.12$ & $\leq 2$ & No \\
\hline FR-247 & A. baumannii & Turkey & $\mathrm{S}$ & $<0.12$ & $\leq 2$ & No \\
\hline FR-248 & A. baumannii & Turkey & $\mathrm{S}$ & $<0.12$ & $\leq 2$ & No \\
\hline N4 & A. baumannii & Switzerland & $\mathrm{S}$ & 0.25 & $\leq 2$ & No \\
\hline N14 & A. baumannii & Switzerland & $\mathrm{S}$ & $<0.12$ & $\leq 2$ & No \\
\hline FR-250 & A. baumannii & Italy & $\mathrm{R}$ & 8 & $>4$ & No \\
\hline FR-252 & A. baumannii & Italy & $\mathrm{R}$ & 64 & $>4$ & No \\
\hline FR-253 & A. baumannii & Spain & $\mathrm{R}$ & 4 & $>4$ & No \\
\hline FR-254 & A. baumannii & Spain & $\mathrm{R}$ & 16 & $>4$ & No \\
\hline FR-255 & A. baumannii & Switzerland & $\mathrm{R}$ & 128 & $>4$ & No \\
\hline FR-256 & A. baumannii & Turkey & $\mathrm{R}$ & 16 & $>4$ & No \\
\hline FR-257 & A. baumannii & Turkey & $\mathrm{R}$ & 8 & $>4$ & No \\
\hline FR-258 & A. baumannii & Turkey & $\mathrm{R}$ & 4 & $>4$ & No \\
\hline FR-259 & A. baumannii & Turkey & $\mathrm{R}$ & 4 & $>4$ & No \\
\hline FR-260 & A. baumannii & Turkey & $\mathrm{R}$ & $>128$ & $>4$ & No \\
\hline FR-261 & A. baumannii & Turkey & $\mathrm{R}$ & 4 & $>4$ & No \\
\hline FR-262 & A. baumannii & Turkey & $\mathrm{R}$ & $>128$ & $>4$ & No \\
\hline ATCC 27853 & Pseudomonas aeruginosa & United States & $\mathrm{S}$ & $<0.12$ & $\leq 2$ & No \\
\hline FR-263 & P. aeruginosa & France & $\mathrm{S}$ & $<0.12$ & $\leq 2$ & No \\
\hline FR-264 & $P$. aeruginosa & France & $\mathrm{S}$ & $<0.12$ & $\leq 2$ & No \\
\hline FR-265 & P. aeruginosa & France & $\mathrm{S}$ & $<0.12$ & $\leq 2$ & No \\
\hline FR-266 & P. aeruginosa & France & $\mathrm{S}$ & $<0.12$ & $\leq 2$ & No \\
\hline FR-267 & P. aeruginosa & France & $\mathrm{S}$ & $<0.12$ & $\leq 2$ & No \\
\hline FR-268 & P. aeruginosa & France & $\mathrm{S}$ & $<0.12$ & $\leq 2$ & No \\
\hline FR-269 & P. aeruginosa & France & $\mathrm{S}$ & $<0.12$ & $\leq 2$ & No \\
\hline FR-270 & P. aeruginosa & France & $\mathrm{S}$ & 0.25 & $\leq 2$ & No \\
\hline FR-271 & P. aeruginosa & France & $\mathrm{S}$ & 0.25 & $\leq 2$ & No \\
\hline FR-274 & $P$. aeruginosa & France & $\mathrm{R}$ & 4 & 8 & No \\
\hline FR-275 & P. aeruginosa & France & $\mathrm{R}$ & 32 & 8 & No \\
\hline FR-276 & P. aeruginosa & France & $\mathrm{R}$ & 32 & $>8$ & No \\
\hline FR-277 & P. aeruginosa & France & $\mathrm{R}$ & 16 & 8 & No \\
\hline FR-278 & $P$. aeruginosa & France & $\mathrm{R}$ & 128 & $>8$ & No \\
\hline FR-279 & P. aeruginosa & France & $\mathrm{R}$ & 8 & 4 & No \\
\hline FR-281 & $P$. aeruginosa & France & $\mathrm{R}$ & 4 & $>8$ & No \\
\hline
\end{tabular}

The colistin-resistant isolates are shaded in gray.

BMD, broth microdilution; MIC, minimum inhibitory concentration; R, resistant; S, susceptible. 
Rapid Polymyxin Acinetobacter

and Pseudomonas tests

The Rapid Polymyxin Acinetobacter and Pseudomonas tests from Elitech Microbiology (www.elitechgroup.com/ france) were performed according the manufacturer instructions. In brief, a standardized suspension of each isolate is prepared using a medium specific to each species. A specific volume of this suspension is then placed in the different wells of the kit containing defined amounts of colistin to obtain final concentrations of 0 (positive control), 2 , and $4 \mathrm{mg} / \mathrm{L}$ for both species, and in addition of $8 \mathrm{mg} / \mathrm{L}$ for $P$. aeruginosa. A supplementary well is used as a negative control in which a suspension with only $\mathrm{NaCl}$ is prepared. After 3-4 hours of incubation at $37^{\circ} \mathrm{C}$, a valid result is obtained when a color shift is observed in the positive control well, and no color shift in the negative control well. The result is then read for each colistin containing well as an MIC reading. For this evaluation, the results of susceptibility/resistance to polymyxins for each isolate were observed after 2 hours every 15 minutes until 4 hours.

\section{Result analysis}

The results obtained with the Rapid Polymyxin tests were compared with those obtained with the reference BMD method. In brief, discrepancies were determined to assess their performance to detect colistin susceptibility. Very major errors (VME) and major errors (ME) corresponding to false-susceptible and false-resistant results, respectively, were calculated as described elsewhere. ${ }^{11,12}$

\section{Results}

A total of 21 A. baumannii and 17 P. aeruginosa isolates were included to evaluate the performances of the Rapid Polymyxin Acinetobacter and Pseudomonas tests (Table 1). All of the nine A. baumannii isolates defined as colistin susceptible according to the result of the BMD method (MICs of colistin ranging from $<0.125$ to $0.25 \mathrm{mg} / \mathrm{L}$ ) were identified as susceptible by the Rapid Polymyxin Acinetobacter test (Table 1). All of the 12 colistin-resistant $A$. baumannii isolates (MICs of colistin ranging from 4 to $>128 \mathrm{mg} / \mathrm{L}$ ) were detected as colistin resistant by the Rapid Polymyxin Acinetobacter test (Table 1). Out of the 10 colistin-susceptible $P$. aeruginosa isolates (MICs of colistin ranging from $<0.125$ to $0.25 \mathrm{mg} / \mathrm{L}$ ), all were found susceptible using the Rapid Polymyxin Pseudomonas test. All of the seven colistin-resistant $P$. aeruginosa isolates (MICs of colistin ranging from 4 to $128 \mathrm{mg} / \mathrm{L}$ ) were identified as colistin resistant by the Rapid Polymyxin Pseudomonas test (Table 1). Interpretation of the results for all isolates was obtained between 3 and 4 hours; no positive result was observed before 3 hours.

\section{Discussion}

Out of the 19 colistin-susceptible A. baumannii and $P$. aeruginosa isolates, the Rapid Polymyxin Acinetobacter test identified correctly all susceptible isolates, hence no $\mathrm{ME}$ (i.e., false resistance) was detected for both tests (specificities of $100 \%)$. Out of the 19 colistin-resistant A. baumannii and $P$. aeruginosa isolates, the Rapid Polymyxin
Acinetobacter and Pseudomonas tests were excellent with no VME (i.e., false susceptibility; sensitivities of $100 \%$ ).

This study showed that the Rapid Polymyxin Acinetobacter and Pseudomonas tests are reliable tools for detecting resistance to colistin in A. baumannii and $P$. aeruginosa. In comparison, BMD systems (Sensititre [ThermoFischer Diagnostics] and UMIC [Biocentric and MicroScan]) have been evaluated and showed VME for the three systems and ME for the MicroScan system. ${ }^{13}$ Moreover, these are the first tests that are available for determination of colistin susceptibility in those species in $<4$ hours. However, other evaluations with a higher number of isolates should be performed to confirm our results as well as at least a multicenter study and its possible interest directly from clinical samples. Although MICs of colistin are only determined in ranges $(\leq 2$, comprised between 2 and $4 \mathrm{mg} / \mathrm{L}$, and $>4 \mathrm{mg} / \mathrm{L}$ for $A$. baumannii and $\leq 2$, comprised between 4 and $8 \mathrm{mg} / \mathrm{L}$, and $>8 \mathrm{mg} / \mathrm{L}$ for $P$. aeruginosa) using those tests, they give results of susceptibility/resistance categorization very rapidly, which is the most important feature with respect to the treatment strategy and may contribute to optimize antibiotic stewardship. Colistin is indeed often used to treat infections caused by MDR A. baumannii and $P$. aeruginosa isolates, mostly remaining susceptible to colistin. ${ }^{14}$ However, the increasing trend of acquired colistin resistance observed in those species, and particularly in A. baumannii, ${ }^{15}$ highlights the interest to use such rapid tests able to efficiently detect that resistance trait.

\section{Acknowledgments}

We thank the Elitech Microbiology company for providing us kits of Rapid Polymyxin Acinetobacter and Pseudomonas tests. We thank Javier Fernandez, Véronique Dubois, and Deniz Güneşer for providing several resistant strains. This work has been funded by the University of Fribourg and by the Swiss National Reference Center for Emerging Antibiotic Resistance, Fribourg, Switzerland. It has also been funded by the Swiss National Science Foundation (projects FNS407240_177381 and FNS-407240_177382).

\section{Disclosure Statement}

No competing financial interests exist.

\section{References}

1. Boucher, H.W., G.H. Talbot, J.S. Bradley, J.E. Edwards, D. Gilbert, L.B. Rice, M. Scheld, B. Spellberg, and J. Bartlett. 2009. Bad bugs, no drugs: no ESKAPE! An update from the Infectious Diseases Society of America. Clin. Infect. Dis. 48:1-12.

2. Vincent, J.-L., J. Rello, J. Marshall, E. Silva, A. Anzueto, C.D. Martin, R. Moreno, J. Lipman, C. Gomersall, Y. Sakr, and K. Reinhart; EPIC II Group of Investigators. 2009. International study of the prevalence and outcomes of infection in intensive care units. JAMA. 302:2323-2329.

3. Wright, H., R.A. Bonomo, and D.L. Paterson. 2017. New agents for the treatment of infections with Gram-negative bacteria: restoring the miracle or false dawn? Clin. Microbiol. Infect. 23:704-712.

4. Shin, B., and W. Park. 2017. Antibiotic resistance of pathogenic Acinetobacter species and emerging combination therapy. J. Microbiol. 55:837-849. 
5. Sievert, D.M., P. Ricks, J.R. Edwards, A. Schneider, J. Patel, A. Srinivasan, A. Kallen, B. Limbago, and S. Fridkin; National Healthcare Safety Network (NHSN) Team and Participating NHSN Facilities. 2013. Antimicrobial-resistant pathogens associated with healthcare-associated infections: summary of data reported to the National Healthcare Safety Network at the Centers for Disease Control and Prevention, 2009-2010. Infect. Control Hosp. Epidemiol. 34:1-14.

6. Willyard, C. 2017. The drug-resistant bacteria that pose the greatest health threats. Nature 543:15.

7. Velkov, T., K.D. Roberts, R.L. Nation, P.E. Thompson, and J. Li. 2013. Pharmacology of polymyxins: new insights into an "old" class of antibiotics. Future Microbiol. 8:711-724.

8. EUCAST. 2016. www.eucast.org/fileadmin/src/media/PDFs/ EUCAST_files/General_documents/Recommendations_for_ MIC_determination_of_colistin_March_2016.pdf

9. Nordmann, P., A. Jayol, and L. Poirel. 2016. Rapid detection of polymyxin resistance in Enterobacteriaceae. Emerg. Infect. Dis. 22:1038-1043.

10. EUCAST. 2016. Breakpoints tables for interpretation of MICs and zone diameters, Version 1.0. www.eucast.org

11. Jayol, A., P. Nordmann, P. Lehours, L. Poirel, and V. Dubois. 2017. Comparison of methods for detection of plasmidmediated and chromosomally encoded colistin resistance in Enterobacteriaceae. Clin. Microbiol. Infect. 24:175-179.

12. International Standard Organization. 2007. Clinical Laboratory testing and in vitro diagnostic test systemssusceptibility testing of infectious agents and evaluation of performance of antimicrobial susceptibility test devices- part 2: evaluation of performance of antimicrobial susceptibility test devices. International Standard ISA 20776-2: 2007. ISO, Geneva.

13. Jayol, A., P. Nordmann, C. André, L. Poirel, and V. Dubois. 2018. Evaluation of three broth microdilution systems to determine colistin susceptibility of Gram-negative bacilli. J. Antimicrob. Chemother. 73:1272-1278.

14. Jones, R.N., M. Flonta, N. Gurler, M. Cepparulo, R.E. Mendes, and M. Castanheira. 2014. Resistance surveillance program report for selected European nations (2011). Diagn. Microbiol. Infect. Dis. 78:429-436.

15. Qureshi, Z.A., L.E. Hittle, J.A. O'Hara, J.I. Rivera, A. Syed, R.K. Shields, A.W. Pasculle, R.K. Ernst, and Y. Doi. 2015. Colistin-resistant Acinetobacter baumannii: beyond carbapenem resistance. Clin. Infect. Dis. 60:1295-1303.

Address correspondence to: Laurent Poirel, PhD Emerging Antibiotic Resistance Unit Medical and Molecular Microbiology

Department of Medicine Faculty of Science and Medicine University of Fribourg Chemin du Musée 18 CH-1700 Fribourg Switzerland

E-mail: laurent.poirel@unifr.ch 